\title{
Tweet, tweet
}

\author{
We conducted an informal survey to learn how scientists are using Twitter. We share some of the responses and \\ discuss why being active on Twitter offers real benefits.
}

A major part of the scientific endeavor is communicating ideas and findings, both to peers and to the broader world for dissemination and discourse. We are now solidly entrenched in the digital age, and online social media platforms have become an important part of daily life for many members of the scientific community, including ourselves as editors of a research journal.

Digital media platforms abound, and scientists use all of them, but in different ways. Twitter is one of the most popular, and we were curious about what draws people to 'science Twitter', how they use it, and whether it might benefit scientists who are not already active on social media in a professional capacity.

We sought interested volunteers (on Twitter, of course!) to fill out a questionnaire and were thrilled to receive 99 responses in one week. The respondents come from within and outside academia and are primarily working in the life sciences, but are diverse in terms of gender, geography, career stage and how long they've been on Twitter. As the responses are exclusively from Twitter users, they are biased toward those who enjoy the platform. As such, what follows is not a scholarly look at social media usage by scientists, but rather a glimpse into the successes and potential pitfalls of science Twitter as perceived by active users. Nevertheless, what we learned is that Twitter has a lot to offer scientists. To see the questionnaire, some of our team's answers, and highlights from the responses, please see our related blog post.

We asked "What attracts you to science Twitter?" and observed a few common themes. People see Twitter as an easy-to-use, one-stop shop for information, ranging from science to world news, that is curated on the basis of their interests. What adds to this convenience is the fact that a great many see their Twitter feed as the fastest and most comprehensive way to get updates on new preprints, publications, innovations, science policy, and grant and job opportunities. Many see it as a critical way in which they stay abreast of what is new and notable and an important venue for serious and constructive scientific conversations.

Somewhat related is that many of the respondents saw Twitter as a necessary venue for self-promotion, bringing immediate attention to their work and raising their visibility in the broader community. One scientist, who defines success by the impact of their work, liked that Twitter solved almost all of their dissemination needs, going so far as to say that Twitter is exactly what the Internet is for. However, using Twitter for self-promotion was also viewed negatively, with some feeling obliged to participate lest they fade into obscurity.

Another overarching trend was that scientists enjoy the networking that can be achieved through Twitter. Many feel more connected to the scientific community at large because they find the platform a great place to meet and stay in touch with scientists across the globe. Many of the respondents said they have developed collaborations through Twitter; advertising postdoctoral positions on Twitter appears especially fruitful, both for job seekers and for the labs hiring.

Once started, many seem to stay for more nuanced reasons, perhaps reflecting a basic human desire to connect with like-minded individuals. Many scientists said they feel supported by individuals experiencing the same trials and successes that they are, highlighting the flexibility of Twitter to meet the diverse needs of scientists at different career stages. They also like celebrating and commiserating on the platform. Most said this is especially true in the midst of the coronavirus pandemic, when they are turning to Twitter both for news and for personal connection while social distancing.

We also asked whether scientists keep their tweeting professional or allow their personal lives into the fold, and nearly three-quarters said they keep it strictly professional. For most, this was because they tweet as part of their job or they are only interested in Twitter for the science. Several voiced concerns about how personal tweets might be perceived by, for example, potential colleagues or hiring committees. One early-career researcher kept things strictly professional while on the job market, but hopes to have a more general approach to tweeting in the future. One prudent piece of advice given was not to tweet anything one would not say in a crowd.

Among those who blend their personal and professional lives, the primary reason was they find it important to show publically that scientists are not robots, but real people with diverse interests. Others tried to keep them separate, but ultimately found it easier to find a blend with which they were happy. Seeing the personal side of scientists was something that came up multiple times as a reason people were drawn to Twitter, and many who choose to only tweet about science do enjoy seeing personal tweets in their feed and following people on Twitter who are outside the scientific community.

Of course, a main downside of Twitter is that it can be a time sink. Most respondents said they log in every day, often multiple times per day, with the typical person spending up to an hour a day on Twitter. Some had clever tricks for limiting their use, like setting a timer or only logging on as a reward for an accomplished task. Although Twitter is often work-related, several noted that being on the site can be perceived as a waste of time.

We are happy to report that fewer than a handful we heard from have been trolled on Twitter, although a number know others who have been trolled or censor their own tweets for fear of being a target. Some suggestions were to unfollow, mute, block and report liberally when faced with hostility and to support fellow scientists who are victims of online trolling.

The responses to our survey were such an abundance of riches that it was impossible to discuss everything in this piece (a big thanks to everyone who helped!). We envision this as the first in a series of editorials on social media in our pages. Other useful resources on science and social media include "A scientist's guide to social media" by Jennifer Heemstra and a Comment entitled "The reward and risk of social media for academics” by Ben Britton, Chris Jackson and Jessica Wade.

Many of us at Nature Methods are active on Twitter, and we recommend that those who haven't experienced it take it for a spin. Please find us @rita_strack, @madhuramukho, @rushingmania, @allisondoerr14, @metricausa and of course@naturemethods.

Published online: 4 June 2020 https://doi.org/10.1038/s41592-020-0860-6 